\title{
No "optimal timing" of renal-replacement therapy in critically ill patients with acute kidney injury
}

\author{
Zhiwei Zhang \\ Division of Nephrology, Department of Medicine, VA Loma Linda Healthcare System and Loma Linda University School of Medicine, Loma \\ Linda, CA, USA \\ Correspondence to: Zhiwei Zhang, MD. VA Loma Linda Healthcare System, Nephrology 111N, 11201 Benton Street, Loma Linda, CA 92357, USA. \\ Email: zhiwei.zhang@va.gov. \\ Provenance: This is an invited Editorial commissioned by Section Editor Wei Liu, MD (Department of Nephrology, The Affiliated Anqing Hospital \\ of Anhui Medical University Anqing, Anqing, China). \\ Comment on: Barbar SD, Clere-Jehl R, Bourredjem A, et al. Timing of Renal-Replacement Therapy in Patients with Acute Kidney Injury and Sepsis. \\ N Engl J Med 2018;379:1431-42.
}

Submitted Nov 13, 2018. Accepted for publication Nov 27, 2018.

doi: $10.21037 / \mathrm{atm} .2018 .11 .62$

View this article at: http://dx.doi.org/10.21037/atm.2018.11.62

Acute kidney injury (AKI) represents a sudden decrease in renal function from a number of disparate causes (1). Using KDIGO (Kidney disease: Improving Global Outcomes) definition, a meta-analysis indicated that the world incidence rates of AKI were approximately $21 \%$ in adults and $33 \%$ in children (2). Acute tubular necrosis (ATN) accounts for the majority cases of AKI especially in critical care setting with mortality rate exceeding 50\% (3). The current medical management of AKI/ATN, however, is limited to supportive care and renal-replacement therapy (RRT) if indicated, while various therapeutic modalities have been attempted without success.

Although there is little controversy for initiation of RRT in critical ill patients with AKI complicated by lifethreatening or medically refractory conditions such as severe hyperkalemia, metabolic acidosis or diuretic-resistant fluid overload with pulmonary edema, the appropriate timing of RRT in other situations remains a subject of debate. The theoretical benefits of RRT (Table 1) must be weighed against its potential drawbacks (Table 2) in the absence of life-threatening complications and early sign of kidney recovery since the renal dysfunction in AKI/ ATN could have high likelihood to recover spontaneously. However, evidence-based studies to guide clinical practice are essentially absent in this area, resulting in wide variations in decision-making process and inconsistent and potentially suboptimal quality of care.
For the past 20 years, several randomized trials focused on various timings of RRT initiation on clinical outcomes in critical ill patients with AKI were completed, including high-profile ELAIN (The Early Versus Late Initiation of Renal Replacement Therapy in Critically III Patients with Acute Kidney Injury) and AKIKI (The Artificial Kidney Initiation in Kidney Injury) trials (4). The ELAIN trial is a single-center randomized trial conducted in Germany, which evaluates the effects of early (initiating RRT at KDIGO stage $2 \mathrm{AKI}$ ) vs. delayed (initiating RRT at KDIGO stage 3 AKI or upon an severe medically refractory complication requiring RRT was present) initiation of RRT in the course of critically ill patients with AKI (5). Among 231 patients enrolled in the study, all patients assigned to early-initiation $(\mathrm{n}=112)$ and majority of patients in the delayed-initiation group ( $n=108 / 119)$ received RRT. The primary outcome of 90-day mortality was significantly better in the earlyinitiation group $(39.3 \%$ vs. $54.7 \%$ in the delayed-initiation group, $\mathrm{P}=0.03)$. Secondary outcomes including less dialysis independence and short duration of RRT, and shortening of hospital stay were also in favor of early initiation of RRT.

The AKIKI trial is a multicenter randomized trial conducted from 31 intensive care units (ICU) in France, which examines the effect of early (initiating RRT immediately after randomization) $v s$. delayed (initiating RRT only upon development of severe medically refractory complications requiring RRT) initiation of RRT in 
Table 1 Theoretical benefits of RRT

Maintenance of homeostasis
Fluid, electrolytes, acid-base
Solute clearance
Uremic control, immunomodulation and clearance of
inflammatory mediators
Facilitation of additional supportive measures
Nutrition, blood products, medications
Limitation of worsening non-renal organ dysfunction

Potential kidney-organ (heart, lung, brain) interactions

$\mathrm{RRT}$, renal-replacement therapy.

Table 2 Theoretical drawbacks of RRT

Dialysis catheter insertion
Bleeding, infection, pneumothorax
Exposure to extracorporeal circuit
Dialyzer reaction or inflammation
Excess loss or clearance nutrients or medications
Micronutrients, trace elements, antibiotics
"latrogenic" hemodynamic insults
May exacerbate AKI and impede kidney repair/recovery
Maladaptive neuro-hormonal response to RRT
May delay kidney recovery
Unnecessary exposure to RRT
Spontaneous kidney recovery
Healthcare costs

RRT, renal-replacement therapy; AKI, acute kidney injury.

620 patients with KDIGO stage 3 AKI who were critically ill but not requiring urgent RRT (6). There was no significant difference in the primary outcome of 60-day mortality between the early $(48.5 \%)$ and delayed (49.7\%) RRT groups $(\mathrm{P}=0.79)$. However, diuresis occurred earlier in the delayed-RRT group and almost half of the patients (49\%) in this group never received RRT. The incidence of catheterrelated infections was also lower in the delayed-RRT group.

Both ELAIN and AKIKI trials are important but their discrepant results create confusion (4). First, the findings from ELAIN trial are difficult to be generalized since it was a single-center trial with primarily surgical patients. Secondly, the ELAIN trial had a very low fragility index of 3 (4), indicating that only 3 more or fewer events in either group would alter the statistical significance so the observed findings from this study should be interpreted carefully. Thirdly, kidney function at the time of RRT initiation was different in the ELAIN trial (KDIGO stage 2 for early group, and KDIGO stage 3 for delayed arm). Although the primary end point of mortality at day 90 was significantly different, but not for mortality at earlier time points (day 28 or day 60 ), implying that a possibility of factors other than the timing of RRT affecting the late mortality in critical ill patients could not be excluded. Finally, the AKIKI trial used a different "timing" threshold (KDIGO stage 3) for initiation of RRT in early group, which actually corresponds to the delayed RRT arm in the ELAIN trial, making comparison between these 2 trials impossible at all.

A meta-analysis of 9 randomized trials completed after year 2002 including both ELAIN and AKIKI was performed to evaluate the effect of timing of RRT initiation on clinical outcomes in critically ill patients with AKI (7). The pooled data did not observe significant between-group difference in the odds ratio for death among patients receiving early (327 of $827,39.5 \%$ ) or delayed (338 of $899,37.6 \%$ ) initiation of RRT $(\mathrm{P}=0.80)$. Thus, additional large multicenter trials are urgently needed to confirm or refute this important finding.

Barbar et al. now report the primary results of IDEALICU (Initiation of Dialysis Early Versus Delayed in the Intensive Care Unit) trial, which took place in 29 ICUs in France (8). The trial involved 488 patients with early stage septic shock (within 48 hours after the start of vasopressor therapy) who had severe AKI classified at the failure stage of RIFLE (The risk, injury, failure, loss, and end-stage kidney disease) system, which is present if a serum creatinine concentration 3 times the baseline level [or $\geq 4 \mathrm{mg} / \mathrm{dL}$ $(\geq 350 \mu \mathrm{mol} / \mathrm{dL})$ accompanied by a rapid increase of $\geq 0.5 \mathrm{mg} / \mathrm{dL}(\geq 44 \mu \mathrm{mol} / \mathrm{dL})$ ], urine output $<0.3 \mathrm{~mL} / \mathrm{kg} / \mathrm{h}$ for 24 hours, or anuria for 12 hours or more. Patients who required urgent RRT before randomization for lifethreatening or medically refractory complications relate to AKI (metabolic acidosis with $\mathrm{pH}<7.15$, hyperkalemia $>6.5 \mathrm{mmol} / \mathrm{L}$ or diuretic-resistant fluid overload with pulmonary edema) were not eligible for the trial.

After a diagnosis of AKI at RIFLE failure-stage was documented, patients were randomly assigned to either early (receiving RRT within 12 hours, 246 patients) or delayed-RRT group (receiving RRT after 48 hours, 242 
patients). Patient assigned to the delayed-RRT group were closely monitored after randomization to detect the occurrence of life-threatening or medically refractory complications related to AKI as described earlier, and RRT was initiated as soon as possible once any of those conditions developed. Meanwhile, patients in the delayedRRT group would not receive RRT if spontaneous renal recovery occurred as defined as a decrease in serum creatinine level and a return of spontaneous urine output to $>1,000 \mathrm{~mL} /$ day (or $>2,000 \mathrm{~mL} /$ day in patients receiving diuretics). The primary outcome was death at 90 days.

Follow-up data were available at 90 days in 477 patients enrolled in the trial. The primary end point of mortality at 90 days was similar in the early (58\%) and the delayed (54\%) RRT groups $(\mathrm{P}=0.38)$. Among patients in the delayed RRT group, $38 \%$ of them never required RRT. The delayed strategy also resulted in significantly larger number of days free of RRT than the early strategy $(\mathrm{P}=0.006)$, but other secondary outcomes including mortality at 28 days and 180 days, number of days free of mechanical ventilation and vasopressors, and length of ICU and hospital stay were not significantly different between the groups. Although the use of RIFLE classification rather than other commonlyused and more sensitive classification systems to define AKI may limit the generalizability, this carefully designed trial provides further clarification to reconcile the discordant findings on the effect of timing of RRT initiation in critically ill patients with AKI.

The clinical presentations for patients in critical care setting are highly variable, and reliable tools to predict the need for RRT are extremely limited. Current evidence did not confirm the theoretical advantage of earlier initiation of RRT (Table 1) in the absence of life-threatening or medically refractory complications. In addition, initiation of RRT too early could unnecessarily expose patients to the potential risks associated with RRT (Table 2). For an individual patient, timing of RRT initiation should be not too early, not too late, but just at the right time. A decision to initiate RRT on daily clinical practice will likely rely on a careful assessment and judicious integration of patient baseline characteristics, comorbid conditions, acuity of diagnosis, disease progression, urgency of complications, involvement of extrarenal organs and, and timely availability of resource and technical expertise. Obviously one size will never fit all. An attempt to protocolize an "optimal timing" of RRT in critically ill patients with AKI based on pre- defined thresholds may be imprecise and impractical.

\section{Acknowledgements}

None.

\section{Footnote}

Conflicts of Interest: The author has no conflicts of interest to declare.

\section{References}

1. Zhang Z. Acute kidney injury. In: Schmitz PG, Martin KJ, editors. Internal Medicine Just the Facts. McGraw-Hill, 2008:687-96.

2. Susantitaphong P, Cruz DN, Cerda J, et al. World incidence of AKI: a meta-analysis. Clin J Am Soc Nephrol 2013;8:1482-93.

3. Liano F, Pascual J. Epidemiology of acute renal failure: a prospective, multicenter, community-based study. Madrid Acute Renal Failure Study Group. Kidney Int 1996;50:811-8.

4. Bagshaw SM, Wald R. Strategies for the optimal timing to start renal replacement therapy in critically ill patients with acute kidney injury. Kidney Int 2017;91:1022-32.

5. Zarbock A, Kellum JA, Schmidt C, et al. Effect of Early vs Delayed Initiation of Renal Replacement Therapy on Mortality in Critically Ill Patients With Acute Kidney Injury: The ELAIN Randomized Clinical Trial. JAMA 2016;315:2190-9.

6. Gaudry S, Hajage D, Schortgen F, et al. Initiation Strategies for Renal-Replacement Therapy in the Intensive Care Unit. N Engl J Med 2016;375:122-33.

7. Gaudry S, Hajage D, Dreyfuss D. Initiation of RenalReplacement Therapy in the Intensive Care Unit. N Engl J Med 2016;375:1901-2.

8. Barbar SD, Clere-Jehl R, Bourredjem A, et al. Timing of Renal-Replacement Therapy in Patients with Acute Kidney Injury and Sepsis. N Engl J Med 2018;379:1431-42.

Cite this article as: Zhang Z. No "optimal timing" of renalreplacement therapy in critically ill patients with acute kidney injury. Ann Transl Med 2018;6(Suppl 2):S112. doi: 10.21037/ atm.2018.11.62 